\title{
Produção e Qualidade de Ovos em Reprodutoras de Frangos de Corte com Horário de Arraçoamento Diferenciado ${ }^{1}$
}

\author{
Valdir Silveira de Avila², Antônio Mário Penz Jr. ${ }^{3}$, Paulo Antônio Rabenschlag de Brum ${ }^{4}$, Antônio \\ Lourenço Guidoni ${ }^{2}$, Paulo Sérgio Rosa ${ }^{5}$, Arlei Coldebella ${ }^{6}$
}

RESUMO - Foi estudada a influência de quatro horários de arraçoamento (único, às 6h30; dual [ $50 \%$ às $6 \mathrm{~h} 30$ e 50\% às $15 \mathrm{~h} 30$ ]; único, às $11 \mathrm{~h}$; e único, às 15h30) sobre a produção e qualidade dos ovos de reprodutoras de corte, alocados ao acaso, em 48 boxes, com 27 fêmeas Arbor Acres e três machos cada, com 12 repetições. As exigências nutricionais e de manejo atenderam as recomendações da linhagem. Estudaram-se as produções de ovos total (PTOTAL), de ninho (PNINHO), limpos (PLIMPO) e quebrados (PQUEMO) em seis coletas (6h30,9h, 11h, 13h30, 15h30 e 17h30); peso médio (PESOVO) e gravidade específica do ovo (GE) agrupados em três coletas (9h, 13h30 e 17h30). Foram utilizados a análise de variância, o teste T de Student para comparação das médias referentes a tratamento e coleta, e a análise de regressão polinomial para idade. Houve efeito do horário de arraçoamento e coleta para todas as variáveis, exceto para PQUEMO, em que não foi evidenciado efeito do horário de arraçoamento. Para os arraçoamentos às 6 h30 e dual, o maior percentual de ovos produzido $(22,78 \pm 0,36 \%$ e $20,26 \pm 0,33 \%$, respectivamente) ocorreu na coleta das $9 \mathrm{~h}$, enquanto, para os arraçoamentos às $11 \mathrm{~h}$ e às $15 \mathrm{~h} 30$, ocorreu na coleta das $13 \mathrm{~h} 30(20,35 \pm 0,39 \%$ e 19,25 $\pm 0,38 \%$, respectivamente). As reprodutoras do sistema dual de arraçoamento e aquelas arraçoadas às $15 \mathrm{~h} 30$ tiveram PESOVO de 71,0 $\pm 0,3 \mathrm{~g}$, que, em qualquer coleta, foi, no mínimo, igual aos demais. As médias de GE foram maiores para as aves arraçoadas às $11 \mathrm{~h}(1,0788 \pm 0,0002)$ e às $15 \mathrm{~h} 30(1,0799 \pm 0,0002)$ que as demais. Ovos de reprodutoras mais velhas e aqueles coletados nas primeiras horas do dia foram mais pesados e apresentaram cascas mais finas. $\mathrm{O}$ sistema de arraçoamento dual apresentou-se como a melhor alternativa na substituição do sistema de alimentação única às $6 \mathrm{~h} 30$.

Palavras-chave: horário de arraçoamento, produção de ovos, qualidade do ovo, reprodutoras de frangos de corte

\section{Performance and Egg Quality Traits of Female Broiler Breeders Submitted to Different Feeding Schedules}

\begin{abstract}
Four feeding schedule were investigated for broiler breeders: at 6:30 am; 50\% feeding at 6:30 am and 50\% at $3: 30 \mathrm{pm}$ (dual); at 11:00 am, and at 3:30 pm. Arbor Acres breeders were randomly assigned to 48 boxes, with 12 replicates of 27 females and three males each. The nutritional levels and management procedures were those recommended for strain. The variables studied were: total egg production, nest, clean and broken egg production, in six collections (6:30 am, 9:00 am, 11:00 am, 1:30 pm, 3:30 pm and 5:30 pm); egg weight and egg specific gravity, grouped in three collections (9:00 am, 1:30 pm and 5:30 pm). Analysis of variance was used. Means were compared using Student $t$ test for traits and collection and polynomial regression analysis for age. The collection and feeding schedule effects were significant for all traits, except for broken eggs, in which feeding schedule was not significant. The maximum egg production was obtained at 9:00 am for feeding schedule at 6:30 am (22.78 $\pm 0.36 \%)$ and dual $(20.26 \pm 0.33 \%)$, and at 1:30 pm for feeding schedule at $11: 00 \mathrm{am}(20.35 \pm 0.39 \%)$ and at $3: 30 \mathrm{pm}(19.25 \pm 0.38 \%)$. Female broiler breeders under the dual and at $3: 30 \mathrm{pm}$ feeding schedule showed egg weight of $71.0 \pm 0.3 \mathrm{~g}$, which was at least equal to the other feeding schedule in any collection. The egg specific gravity for feeding schedule at 11:00 am (1.0788 \pm 2$)$ and 3:30 pm $(1.0799 \pm 2)$ were higher than the other feeding schedule. Eggs from older female broiler breeders and eggs collected in the first hours of the day were heavier and had thinner eggshell than those from younger females and eggs collected later in the day. The dual feeding schedule could be an alternative to feeding schedule at 6:30.
\end{abstract}

Key Words: egg production, egg quality, feeding schedule, female broiler breeders

\section{Introdução}

Na produção industrial de ovos para incubação, buscam-se práticas para concentrar a postura nos ninhos, evitar a postura na cama e, conseqüentemente, a presença de ovos sujos. Tanto o manejo dos ninhos na pré-postura como a utilização de um número adequado de coletas diárias, contribuem na obtenção de

\footnotetext{
${ }^{1}$ Parte da tese apresentada à UFGRS, pelo primeiro autor, para obtenção do título de Doutor em Zootecnia, com apoio financeiro do CNPq 2 Embrapa Suínos e Aves - Eng. Agr., DSc. E.mail: vavila@cnpsa.embrapa.br, antlog@cnpsa.embrapa.br

3 Universidade Federal do Rio Grande do Sul - Eng. Agr., PhD. E.mail: ampenz@conex.com.br

${ }^{4}$ Embrapa Suínos e Aves - Méd. Vet., DSc. E.mail: pbrum@cnpsa.embrapa.br. Bolsista do CNPq.

5 Embrapa Suínos e Aves - Zootec., MSc. E.mail: prosa@cnpsa.embrapa.br

${ }^{6}$ Embrapa Suínos e Aves - Méd. Vet., DSc. E.mail: arlei@cnpsa.embrapa.br
} 
ovos limpos e com menor grau de contaminação. Além disso, o peso do ovo e a qualidade da casca (gravidade específica) são fatores importantes na produção do ovo fértil. Considera-se que maior gravidade específica resulta em melhor qualidade de casca e, conseqüentemente, em ovos mais apropriados para incubação. Salienta-se, no entanto, a importância da relação entre peso do ovo e gravidade específica, em que o peso do ovo aumenta e a gravidade específica diminui com a idade das reprodutoras.

Cave (1981) e Bootwalla et al. (1983) colocam em dúvida se o esquema de alimentação única pela manhã atende à demanda por nutrientes, especialmente no momento da formação da casca do ovo. Cave (1981), trabalhando com matrizes pesadas, constatou que a alimentação duas ou três vezes ao dia permitiu melhora no peso e na produção de ovos e maior eficiência na utilização do alimento.

Farmer \& Roland Sr (1983) e Bootwalla et al. (1989) relataram que os diferentes horários de alimentação influenciaram o ciclo de formação do ovo e os níveis de cálcio e fósforo do sangue no momento da calcificação e que o horário de maior necessidade de cálcio, para a formação da casca do ovo, ocorreu no período noturno. No entanto, Brake (1988) observou que tanto a produção quanto o peso do ovo não foram influenciados pelo horário da alimentação.

De acordo com Summers (1987) e Rutz (1992), o catabolismo do excesso de proteína em ácido úrico produz mais calor corporal, ocasionando estresse adicional ao das elevadas temperaturas. Aves alimentadas às $6 \mathrm{~h}$ apresentaram maior incremento calórico cinco horas após a refeição que aquelas alimentadas às 14h (Wilson et al., 1989). Incremento calórico promove alta ofegação e perda de $\mathrm{CO}_{2}$, podendo tornar o problema ainda mais grave para as reprodutoras em virtude da temperatura ambiental. Como recurso para perda de calor ou redução da temperatura corporal, surgem problemas na produção de ovos decorrentes da falta do $\mathrm{CO}_{2}$ na principal reação de formação do carbonato de cálcio, necessário para a calcificação da casca do ovo.

Neste contexto, realizou-se este experimento com o objetivo de associar o horário de fornecimento do alimento às aves com a produção de ovos por coleta diária, peso e gravidade específica do ovo em reprodutoras de frangos de corte.

\section{Material e Métodos}

Utilizou-se um aviário experimental nas dependências da Embrapa Suínos e Aves para alojar, a partir das 20 semanas de idade, 1.296 fêmeas da linhagem Arbor Acres e 144 machos de outra linhagem comercial, distribuindo-se 27 fêmeas e três machos por boxe, no período de 24 a 66 semanas de idade. Para se evitar o estresse, a ração (peletizada e posteriormente moída) a ser fornecida no dia seguinte era pesada e os comedouros levantados logo após a refeição do dia. Para as fêmeas, a quantidade de ração diária foi baseada no peso corporal e na produção de ovos, e para os machos, tomou-se como base apenas o peso corporal. O peso corporal foi obtido em intervalos de duas semanas, utilizando-se a média de quatro boxes por tratamento. Os tratamentos consistiram de quatro horários de fornecimento do alimento (arraçoamento único, às $6 \mathrm{~h} 30$; arraçoamento dual (50\% às $6 \mathrm{~h} 30$ e $50 \%$ às $15 \mathrm{~h} 30)$; arraçoamento único, às $11 \mathrm{~h}$; e arraçoamento único, às 15h30), iniciados a partir da $20^{\mathrm{a}}$ semana, para adaptação das aves. Para se evitar cama úmida, houve restrição de água durante o período experimental, que foi fornecida de $6 \mathrm{~h} 30$ às $9 \mathrm{~h}$, de $11 \mathrm{~h}$ às $13 \mathrm{~h} 30$ e de $15 \mathrm{~h} 30$ às $18 \mathrm{~h}$. Eventualmente, em dias quentes, não houve restrição. Foram adotados os seguintes períodos para cada tipo de ração: pré-postura, de 18 a 23 semanas, postura I, de 24 a 47 semanas e postura II, de 48 a 66 semanas de idade. A debicagem, o programa de luz e as exigências nutricionais atenderam as recomendações do manual da linhagem Arbor Acres (Sadia, 1993). A porcentagem média dos ingredientes das dietas experimentais das reprodutoras encontram-se na Tabela 1 .

Neste trabalho, avaliou-se a classificação dos ovos produzidos em seis coletas diárias, realizadas às $6 \mathrm{~h} 30$, 9h, 11h, 13h30, 15h30 e 17h30.

Em cada boxe, a porcentagem total de ovos (PTOTAL) por coleta foi obtida pelo quociente entre o número total de ovos da coleta da semana e o somatório das reprodutoras sobreviventes em cada dia durante a semana. A porcentagem total de ovos foi calculada pela média das produções durante o período de 25 a 66 semanas. O cálculo dos percentuais de produção para ovos no ninho (PNINHO), limpos (PLIMPO) e quebrados (PQUEMO) obedeceu aos mesmos critérios da variável PTOTAL. Os ovos quebrados e membranosos foram agrupados na variável PQUEMO. Como a porcentagem de ovos de cama é complemento dos ovos de ninho e a porcentagem de ovos sujos é complemento 
Tabela 1 - Composição percentual e calculada da dieta

Table 1 - Calculated (\%) composition of the diet

\begin{tabular}{|c|c|c|c|}
\hline $\begin{array}{l}\text { Ingrediente } \\
\text { Ingredient }\end{array}$ & $\begin{array}{c}\text { Pré - postura } \\
\text { Pre-breeder }\end{array}$ & $\begin{array}{c}\text { Postura I } \\
\text { Breeder I }\end{array}$ & $\begin{array}{c}\text { Postura II } \\
\text { BreederII }\end{array}$ \\
\hline Milho (Corn) & 62,89 & 67,40 & 65,42 \\
\hline Farelo de soja (Soybean meal) & 21,15 & 20,13 & 18,15 \\
\hline Farelo de trigo (Wheat meal) & 9,56 & 1,51 & 5,63 \\
\hline Calcário (Limestone) & 4,18 & 8,28 & 8,22 \\
\hline Fosfato bicálcico (Dicalcium phosphate) & 1,38 & 1,71 & 1,64 \\
\hline Premix vitamínico ${ }^{2}$ (Vitamin premix) & - & 0,10 & 0,10 \\
\hline Premix mineral $^{3}$ (Mineral premix) & 0,15 & 0,15 & 0,10 \\
\hline Dl-Metionina (Dl-Methionine) & 0,16 & 0,20 & 0,15 \\
\hline L-Lisina $(L-L y s i n e)$ & - & 0,02 & 0,007 \\
\hline Cloreto de colina $60 \%$ (Choline chloride $60 \%$ ) & - & 0,08 & 0,088 \\
\hline Vermífugo (Anti-helmintic) & - & - & 0,075 \\
\hline PB (\%) (Crude protein \%) & 16,50 & 15,00 & 14,50 \\
\hline $\mathrm{EM}(\mathrm{kcal} / \mathrm{kg})(\mathrm{ME} \mathrm{kcal} / \mathrm{kg})$ & 2800 & 2800 & 2750 \\
\hline $\mathrm{Ca}(\%)($ Calcium \%) & 1,70 & 3,10 & 3,20 \\
\hline $\mathrm{P}$ disponível (\%) (Available phosphorus \%) & 0,37 & 0,40 & 0,40 \\
\hline P total (\%) (Total phosphorus \%) & 0,64 & 0,61 & 0,63 \\
\hline Metionina (\%) (Methionine \%) & 0,37 & 0,40 & 0,35 \\
\hline Met + Cis $(\%)($ Meth + Cys \%) & 0,65 & 0,65 & 0,60 \\
\hline Lisina (\%) (Lysine \%) & 0,80 & 0,75 & 0,70 \\
\hline Triptofano (\%) (Tryptophan \%) & 0,30 & 0,26 & 0,25 \\
\hline
\end{tabular}

${ }_{1}^{1}$ Premix vitamínico (Vitamin premix): Composição por kg de ração (composition per kilogram of diet) 10.000 UI Vit. A; 2.500 UI Vit. D3; 30 UI Vit. E; 2,5 mg Vit. K3; 2,5 mg Vit.B1 (thiamin); 8 mg Vit. B2 (riboflavin); 4 mg Vit. B6; 0,015 mg Vit B12; 45mg Ác. nicotínico (Nicotinic acid);15mg Ác. pantotênico (Pantothenic acid); $1400 \mathrm{mg}$ Colina (Choline); 0,20 mg Biotina (Biotin); 1,5 mg Ác. Fólico (Folic acid); $200 \mathrm{mg}$ B.H.T - Antioxidante (antioxidant).

2 Premix vitamínico (Vitamin premix): Composição por kg de ração (composition per kilogram the diet) 12.000 UI Vit. A; 3.600 UI Vit. D3; 35 UI Vit. E; 3 mg Vit. K3; 2,5 mg Vit. B1 (thiamin); 8 mg Vit. B2 (riboflavin); 5 mg Vit. B6; 0,020 mg Vit B12; 40 mg Ác. nicotínico (Nicotinic acid); $12 \mathrm{mg} \mathrm{Ác.} \mathrm{Pantotênico} \mathrm{(pantothenic} \mathrm{acid);} \mathrm{0,20} \mathrm{mg} \mathrm{Biotina} \mathrm{(Biotin);} \mathrm{1,5} \mathrm{mg} \mathrm{Ác.} \mathrm{fólico} \mathrm{(Folic} \mathrm{acid).}$

${ }^{3}$ Premix mineral (Mineral premix): Composição por kg de ração (composition per kilogram of diet): 70 mg manganês (manganese); 75 mg zinco (zinc); $40 \mathrm{mg}$ ferro (iron); $8 \mathrm{mg}$ cobre (copper); $0,5 \mathrm{mg}$ iodo (iodine); $0,13 \mathrm{mg}$ selênio (selenium); $132 \mathrm{mg}$ terramicina (terramycin); $250 \mathrm{mg}$ anticoccidiano amprólio (amprolium anticoccidial).

dos ovos limpos em relação à porcentagem total de ovos produzidos, foram apresentadas e discutidas apenas as variáveis porcentagem de ovos de ninho e porcentagem de ovos limpos.

Considerou-se como variáveis caracterizadoras da qualidade do ovo incubável o peso e a gravidade específica.Para avaliar o peso médio do ovo (PESOVO) e a gravidade específica (GE), foram utilizados todos os ovos produzidos em cada boxe, nos dois últimos dias das semanas $28,32,36,40,44,48,52$, 56, 60 e 65. As coletas foram redefinidas para três, após terem sido reagrupadas duas a duas, obedecendo a seqüência natural inicialmente estabelecida.
Para determinação da GE, após pesagem, os ovos foram colocados dentro de uma cesta e mergulhados em 10 baldes com soluções de densidade diferentes, calibradas por um densímetro, variando a concentração de 1,062 a 1,098, com incrementos de 0,004 g/mL. Este procedimento foi realizado para cada boxe, efetuando-se, seqüencialmente, a avaliação para todas as coletas dentro do mesmo, antes de iniciar as avaliações no próximo boxe.

$\mathrm{Na}$ análise das variáveis PTOTAL, PNINHO, PLIMPO, PQUEMO, PESOVO e GE, adotou-se o procedimento GLM do SAS (1996) envolvendo o seguinte modelo para parcelas sub-subdividida: 


$$
\begin{gathered}
y_{i j l k}=\mu+t_{i}+e_{i j}+c_{1}+c t_{i l}+e_{i j l}+s_{k}+t_{i k}+ \\
c_{1 k}+t s_{i l k}+e_{i j l k}
\end{gathered}
$$

em que $\mathrm{i}=1,2,3,4$ tratamentos; $\mathrm{j}=1, \ldots, 12$ boxe; $1=$ $1,2, \ldots, \mathrm{C}$ coletas diárias; $\mathrm{k}=1, \ldots, \mathrm{K}$ semanas de avaliação, de modo que: $\mathrm{y}_{\mathrm{ijlk}}$ é o valor da resposta pertencente ao boxe ij na coleta 1 da semana $\mathrm{k}$; $\mu$, a média da resposta no experimento; $t_{i}$, o efeito do tratamento $\mathrm{i} ; \mathrm{e}_{\mathrm{ij}}$, o erro experimental suposto seguir a distribuição normal de média 0 e variância constante $\mathrm{s}^{2} ; \mathrm{c}_{1}$, o efeito de coleta; $\mathrm{ct}_{\mathrm{il}}$, o efeito da interação tratamento $\mathrm{i}$ x coleta $1 ; \mathrm{e}_{\mathrm{ij}}$, o erro de avaliação decorrente da coleta, suposto seguir a distribuição normal de média zero e variância constante $\sigma_{\mathrm{c}}{ }^{2} ; \mathrm{s}_{\mathrm{k}}$, o efeito de semana $\mathrm{k}$; $\mathrm{ts}_{\mathrm{ik}}$, o efeito da interação tratamento i x semana $\mathrm{k}$; $\mathrm{cs}_{1 \mathrm{k}}$, o efeito de interação coleta $1 \mathrm{x}$ semana $\mathrm{k}$; tcs $_{\mathrm{ilk}}$, o efeito de interação tratamento i x coleta 1 x semana $\mathrm{k}$; e $e_{i j l k}$, o erro de avaliação semanal suposto seguir a distribuição normal de média zero e variância constante $\sigma_{\mathrm{s}}{ }^{2}$. O erro experimental $\mathrm{e}_{\mathrm{ij}}$ é o termo usado para testar hipóteses sobre o efeito de tratamentos; $\mathrm{e}_{\mathrm{ij}}$, o termo usado para testar hipóteses sobre coletas e interação tratamentos x coletas; $e_{i j l k}$, o termo usado para testar hipóteses sobre efeito de semanas e as interações tratamentos $x$ semanas, coletas $\mathrm{x}$ semanas e tratamentos $\mathrm{x}$ coletas $\mathrm{x}$ semanas.

As médias dos tratamentos e das coletas foram comparadas pelo teste $\mathrm{T}$ de Student, enquanto, para a idade das reprodutoras, utilizou-se análise de regressão polinomial.

A inclusão completa das interações duplas e tripla entre os fatores tratamentos, semanas e coletas, no modelo usado, teve como finalidade principal purificar o erro experimental, para aumentar a eficiência do experimento.

Como a interação coletas x semanas e a interação tripla tratamentos $\mathrm{x}$ coletas $\mathrm{x}$ semanas são desprovidas de qualquer interesse prático, ambas não foram desdobradas. Deu-se ênfase aos efeitos principais dos fatores envolvidos e às interações de tratamentos com coletas e de tratamentos com semanas.

\section{Resultados e Discussão}

Houve efeito $(\mathrm{p} \leq 0,05)$ para as variáveis PTOTAL, PNINHO e PLIMPO em relação aos fatores tratamentos, coletas, semanas e às interações entre os fatores.

Para a variável PQUEMO, não foi evidenciado efeito de tratamentos e de interação tratamentos $\mathrm{x}$ semanas. Este resultado contraria os relatos de Robinson et al. (1995), que constataram alta incidência de ovos com casca deformada quando trabalharam com aves alimentadas à vontade.

As interações tratamentos e coletas para PTOTAL, PNINHO e PLIMPO foram semelhantes entre si e seus percentuais médios por coleta encontram-se na Tabela 2. Consta na Figura 1 ilustração gráfica considerando tratamento e coleta para PTOTAL; as demais variáveis apresentaram comportamento semelhante.

Com exceção de ovos quebrados, nas coletas das $9 \mathrm{~h}$ e $11 \mathrm{~h}$, as aves com arraçoamento único às $6 \mathrm{~h} 30$ apresentaram comportamento diferente e superior $(\mathrm{p} \leq 0,05)$ àquelas dos outros tratamentos. Verificouse que os percentuais de produção de ovos dos demais tratamentos foram deslocados para as coletas mais tardias em virtude da interferência do horário de arraçoamento.

As aves que receberam todo o alimento às $6 \mathrm{~h} 30$, ou parte às 6 h30 (dual) tiveram o maior percentual de produção de ovos coletado às $9 \mathrm{~h}$, enquanto aquelas que receberam todo o alimento às 11 h e às $15 \mathrm{~h} 30$ apresentaram o maior percentual de produção de ovos coletados às $13 \mathrm{~h} 30$, demonstrando que o horário de arraçoamento influencia no horário de postura das aves das aves (Figura 1).

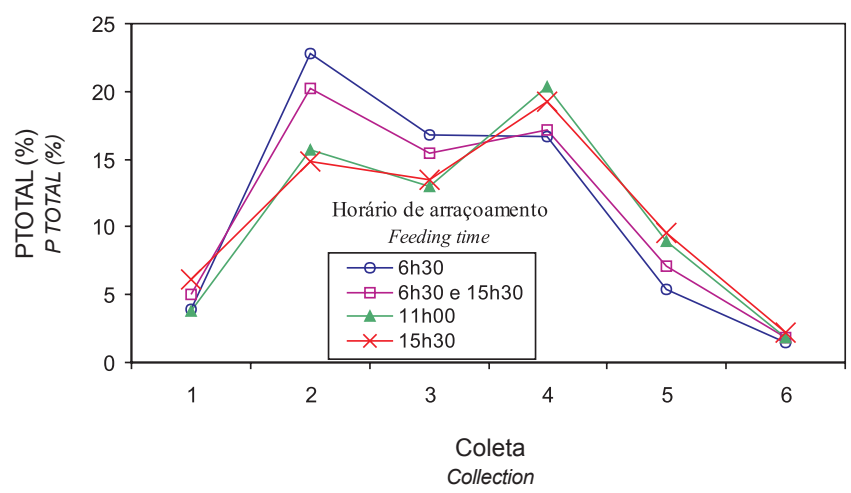

Figura 1 - Perfil do percentual de produção total de ovos (PTOTAL) referente a seis coletas em reprodutoras de frangos de corte, de acordo com o horário de arraçoamento.

Figure 1 - Percentage of total egg prodution (PTOTAL) for six collections in female broiler breeders, according to feeding time. 
Tabela 2 - Percentuais médios de produção total de ovos, ovos de ninho, ovos limpos e ovos quebrados nos diferentes horários de arraçoamento e de coleta em reprodutoras de frangos de corte

Table 2 - Percentage of total eggs, nested eggs, clean eggs and broken eggs (\%) at different feeding and collection time in female broiler breeders

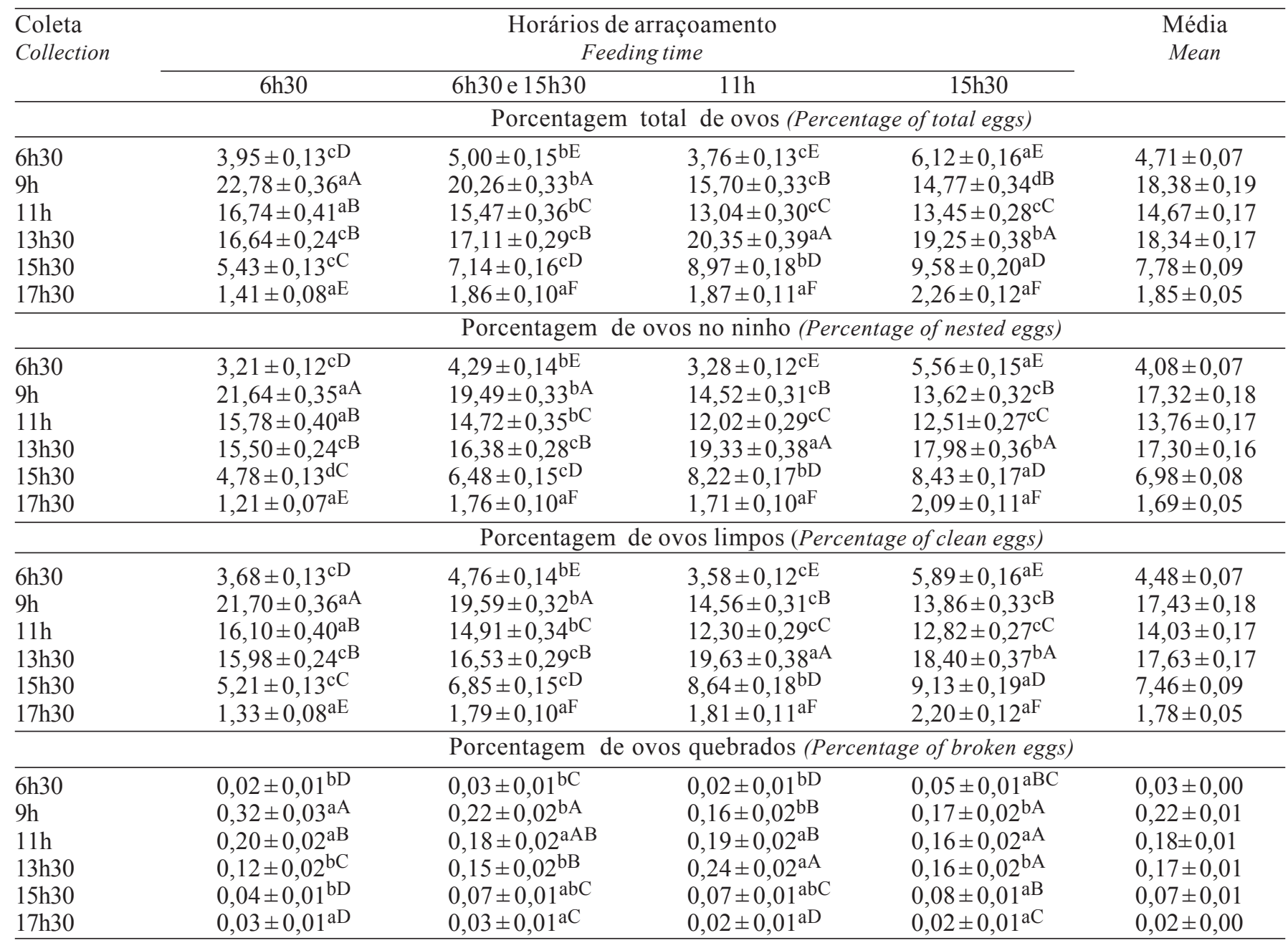

Médias seguidas de letras minúsculas distintas na mesma linha e maiúsculas na mesma coluna diferem significativamente pelo teste $t$ $(\mathrm{p} \leq 0,05)$.

Means followed by different small letters within a row and capital letters in the same column are statistically different ( $p<.05)$ by "t" test.

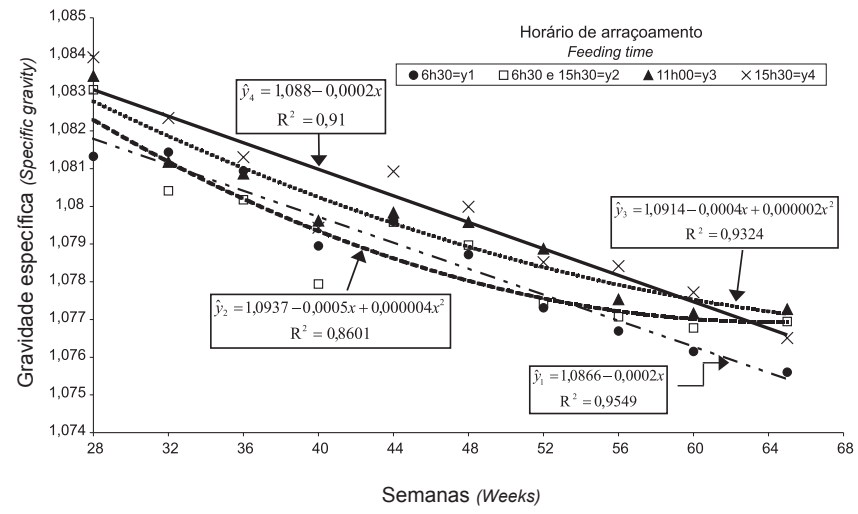

Figura 2 -Perfil da gravidade específica do ovo de acordo com o horário de arraçoamento.

Figure 2 - Egg specific gravity, according to feeding time.
A produção de ovos registrada na primeira coleta é influenciada pelo grupo de aves que realizaram a postura após a última coleta do dia anterior, ou seja, é maior para aves com arraçoamento às $15 \mathrm{~h} 30$ e dual, possivelmente por terem recebido pelo menos parte do alimento mais tarde, o que está de acordo com Samara et al. (1996), que constataram produção de 59,2\% dos ovos antes das $14 \mathrm{~h}$ para as reprodutoras alimentadas às $7 \mathrm{~h}$ e de $53 \%$ para aquelas alimentadas às $18 \mathrm{~h}$, caracterizando atraso na postura desse grupo de aves.

As médias para tratamento dentro de coleta e para tratamento dentro de semana, para PESOVO e GE podem ser observadas nas Tabelas 3 e 4 , respectivamente. Houve interações dupla e tripla $(\mathrm{p} \leq 0,05)$ entre tratamen- 
Tabela 3 - Médias do peso do ovo e da gravidade específica por coleta nos diferentes horários de arraçoamento Table 3 - Mean egg weight and specific gravity by feeding and collection time

\begin{tabular}{|c|c|c|c|c|c|}
\hline \multirow{3}{*}{$\begin{array}{l}\text { Coleta } \\
\text { Collection }\end{array}$} & \multirow{2}{*}{\multicolumn{4}{|c|}{$\begin{array}{l}\text { Horários de arraçoamento } \\
\text { Feeding time }\end{array}$}} & \multirow{3}{*}{$\begin{array}{l}\text { Média } \\
\text { Mean }\end{array}$} \\
\hline & & & & & \\
\hline & $6 h 30$ & $6 h 30$ e $15 h 30$ & $11 \mathrm{~h}$ & $15 \mathrm{~h} 30$ & \\
\hline $9 \mathrm{~h}$ & $70,3 \pm 0,5^{\mathrm{bA}}$ & $71,4 \pm 0,6^{\mathrm{aA}}$ & $71,3 \pm 0,6^{\mathrm{abA}}$ & $70,9 \pm 0,5^{\mathrm{abA}}$ & $71,0 \pm 0,3$ \\
\hline $13 \mathrm{~h} 30$ & $69,4 \pm 0,5^{\mathrm{bB}}$ & $70,9 \pm 0,5^{\mathrm{abAB}}$ & $69,7 \pm 0,6^{\mathrm{bB}}$ & $71,1 \pm 0,6^{\mathrm{aA}}$ & $70,3 \pm 0,3$ \\
\hline $17 \mathrm{~h} 30$ & $69,8 \pm 0,5^{\mathrm{bAB}}$ & $70,6 \pm 0,6^{\mathrm{aB}}$ & $69,2 \pm 0,6^{\mathrm{bB}}$ & $70,9 \pm 0,5^{\mathrm{aA}}$ & $70,1 \pm 0,3$ \\
\hline \multicolumn{6}{|c|}{ Gravidade específica $(\mathrm{g} / \mathrm{mL}) *($ Specific gravity, $\mathrm{g} / \mathrm{mL}) *$} \\
\hline $9 \mathrm{~h}$ & $10767 \pm 3^{\mathrm{bC}}$ & $10767 \pm 2^{\mathrm{bC}}$ & $10783 \pm 3^{\mathrm{aC}}$ & $10782 \pm 3^{\mathrm{aC}}$ & $10775 \pm 1$ \\
\hline $13 \mathrm{~h} 30$ & $10783 \pm 2^{\mathrm{bB}}$ & $10784 \pm 2^{\mathrm{bB}}$ & $10793 \pm 2^{\mathrm{aB}}$ & $10794 \pm 2^{\mathrm{aB}}$ & $10789 \pm 1$ \\
\hline $17 \mathrm{~h} 30$ & $10810 \pm 4^{\mathrm{bA}}$ & $10815 \pm 3^{\mathrm{bA}}$ & $10809 \pm 3^{\mathrm{bA}}$ & $10821 \pm 3^{\mathrm{aA}}$ & $10814 \pm 2$ \\
\hline Média (Mean) & $10787 \pm 2$ & 107882 & $10795 \pm 2$ & 107992 & $10792 \pm 1$ \\
\hline
\end{tabular}

*Valores multiplicados por 10.000. (Values multiplied by 10,000).

Médias seguidas de letras minúsculas distintas na mesma linha e maiúsculas na mesma coluna diferem significativamente pelo teste $\mathrm{T}(\mathrm{p} \leq 0,05)$.

Means followed by different small letters within a row and capital letters in the same column are statistically different $(p<0.05)$ by "t" test.

Tabela 4 - Médias para peso e gravidade específica do ovo por semana para as reprodutoras nos diferentes horários de arraçoamento

Table 4 - Means of egg weight and specific gravity of female broiler breeds by age in weeks, as influenced by different feeding times

\begin{tabular}{|c|c|c|c|c|c|}
\hline \multirow{3}{*}{$\begin{array}{l}\text { Semanas } \\
\text { Weeks }\end{array}$} & \multirow{2}{*}{\multicolumn{4}{|c|}{$\begin{array}{l}\text { Horários de arraçoamento } \\
\text { Feeding time }\end{array}$}} & \multirow{3}{*}{$\begin{array}{l}\text { Média } \\
\text { Mean }\end{array}$} \\
\hline & & & & & \\
\hline & $6 h 30$ & $6 \mathrm{~h} 30$ e $15 \mathrm{~h} 30$ & $11 \mathrm{~h} 00$ & $15 \mathrm{~h} 30$ & \\
\hline \multicolumn{6}{|c|}{ Peso do ovo (g) (Egg weight, g) } \\
\hline 28 & $57,9 \pm 0,8$ & $58,1 \pm 0,4$ & $56,4 \pm 0,6$ & $58,5 \pm 0,7$ & $57,7 \pm 0,3$ \\
\hline 32 & $63,9 \pm 0,3$ & $65,0 \pm 0,4$ & $63,4 \pm 0,5$ & $64,9 \pm 0,3$ & $64,3 \pm 0,2$ \\
\hline 36 & $66,4 \pm 0,3$ & $67,6 \pm 0,3$ & $66,5 \pm 0,4$ & $67,0 \pm 0,3$ & $66,9 \pm 0,2$ \\
\hline 40 & $70,1 \pm 0,4$ & $70,9 \pm 0,4$ & $70,3 \pm 0,4$ & $70,5 \pm 0,2$ & $70,4 \pm 0,2$ \\
\hline 44 & $70,5 \pm 0,3$ & $72,4 \pm 0,4$ & $71,4 \pm 0,4$ & $72,2 \pm 0,3$ & $71,6 \pm 0,2$ \\
\hline 48 & $72,8 \pm 0,4$ & $73,9 \pm 0,4$ & $73,0 \pm 0,4$ & $73,8 \pm 0,4$ & $73,4 \pm 0,2$ \\
\hline 52 & $73,3 \pm 0,4$ & $74,9 \pm 0,4$ & $73,7 \pm 0,4$ & $74,5 \pm 0,4$ & $74,1 \pm 0,2$ \\
\hline 56 & $73,4 \pm 0,3$ & $75,2 \pm 0,4$ & $74,5 \pm 0,5$ & $75,1 \pm 0,5$ & $74,6 \pm 0,2$ \\
\hline 60 & $74,1 \pm 0,3$ & $76,1 \pm 0,4$ & $74,6 \pm 0,4$ & $75,7 \pm 0,4$ & $75,1 \pm 0,2$ \\
\hline 65 & $75,1 \pm 0,3$ & $76,0 \pm 0,4$ & $75,8 \pm 0,4$ & $76,1 \pm 0,5$ & $75,7 \pm 0,2$ \\
\hline Média (Mean) & $69,8 \pm 0,3^{\mathrm{b}}$ & $71,0 \pm 0,3^{\mathrm{a}}$ & $70,1 \pm 0,3^{\mathrm{b}}$ & $71,0 \pm 0,3$ & $70,5 \pm 0,2$ \\
\hline \multicolumn{6}{|c|}{ Gravidade específica $(\mathrm{g} / \mathrm{mL}) *($ Specific gravity, $g / m L) *$} \\
\hline 28 & $10813 \pm 7^{b}$ & $10831 \pm 5^{\mathrm{ab}}$ & $10835 \pm 5^{a}$ & $10840 \pm 7^{a}$ & $10829 \pm 3$ \\
\hline 32 & $10814 \pm 5^{\mathrm{ab}}$ & $10804 \pm 4^{\mathrm{c}}$ & $10812 \pm 3^{b c}$ & $10823 \pm 3^{a}$ & $10813 \pm 2$ \\
\hline 36 & $10809 \pm 4^{\mathrm{ab}}$ & $10802 \pm 5^{b}$ & $10809 \pm 4^{\mathrm{ab}}$ & $10813 \pm 4^{\mathrm{a}}$ & $10808 \pm 2$ \\
\hline 40 & $10790 \pm 4^{\mathrm{ab}}$ & $10779 \pm 4^{b}$ & $10796 \pm 4^{\mathrm{a}}$ & $10794 \pm 4^{\mathrm{a}}$ & $10790 \pm 2$ \\
\hline 44 & $10796 \pm 4^{b}$ & $10796 \pm 5^{b}$ & $10798 \pm 4^{b}$ & $10809 \pm 5^{a}$ & $10800 \pm 2$ \\
\hline 48 & $10787 \pm 5^{b}$ & $10790 \pm 5^{\mathrm{ab}}$ & $10796 \pm 4^{\mathrm{ab}}$ & $10800 \pm 5^{a}$ & $10793 \pm 2$ \\
\hline 52 & $10773 \pm 4^{b}$ & $10774 \pm 4^{b}$ & $10789 \pm 5^{\mathrm{a}}$ & $10785 \pm 5^{a}$ & $10780 \pm 2$ \\
\hline 56 & $10767 \pm 5^{b}$ & $10771 \pm 6^{b}$ & $10775 \pm 5^{\mathrm{ab}}$ & $10784 \pm 6^{\mathrm{a}}$ & $10774 \pm 3$ \\
\hline 60 & $10762 \pm 5^{b}$ & $10768 \pm 5^{\mathrm{ab}}$ & $10772 \pm 5^{\mathrm{ab}}$ & $10777 \pm 5^{\mathrm{a}}$ & $10770 \pm 3$ \\
\hline 65 & $10756 \pm 5^{b}$ & $10769 \pm 5^{\mathrm{a}}$ & $10773 \pm 4^{\mathrm{a}}$ & $10765 \pm 5^{\mathrm{ab}}$ & $10766 \pm 2$ \\
\hline Média (Mean) & $10787 \pm 2$ & $10788 \pm 2$ & $10795 \pm 2$ & $10799 \pm 2$ & $10792 \pm 1$ \\
\hline
\end{tabular}

* Valores multiplicados por 10.000. (Values multiplied by 10,000).

Médias com letras distintas na mesma linha diferem significativamente pelo teste $T(p \leq 0,05)$.

Means with different letters within a row are statistically different $(p \leq 0.05)$ by " $t$ " test. 
tos, coletas e semanas, exceto para tratamentos vs semanas, em relação ao PESOVO, e para tratamentos vs coletas vs semanas, em relação a GE.

Para PESOVO, os tratamentos ocasionaram comportamento diferente em cada coleta, entretanto, as aves que receberam o arraçoamento dual e aquelas que receberam o arraçoamento único às $15 \mathrm{~h} 30$ apresentaram, em qualquer coleta, o PESOVO no mínimo igual aos demais, justificando a maior média geral desses tratamentos. Resultados semelhantes foram constatados por Bootwalla et al. (1983), Farmer \& Roland Sr (1983), Yannakopoulos \& Tserveni-Gousi(1987)e Brake (1988). Este aumento no PESOVO pode ter decorrido da maior eficiência na utilização do alimento. Por sua vez, as aves dos dois tratamentos que receberam todo o alimento pela manhã não foram favorecidas com a mesma intensidade, possivelmente em razão da conhecida correlação negativa entre produção e PESOVO, o que pode ter afetado as aves alimentadas às $6 \mathrm{~h} 30$. $\mathrm{O}$ estresse pelo calor, resultante das temperaturas iguais ou superiores a $30^{\circ} \mathrm{C}$ na maioria das semanas, e o calor metabólico podem ter exercido maior influência sobre as aves alimentadas às 11h. Entretanto, Harms (1991b) não identificou diferença $(p \leq 0,05)$ no PESOVO quando alimentou matrizes de frangos de corte às $8 \mathrm{~h}$ e ou $16 \mathrm{~h}$ no período de 52 a 62 semanas de idade.

O PESOVO decresceu entre a coleta de $9 \mathrm{~h}$ e a de $17 \mathrm{~h} 30$, indicando que ovos coletados nas primeiras horas do dia tendem a ser mais pesados, confirmando os relatos de Brake (1985), que constatou que o PESOVO foi maior quando a postura ocorreu antes das 9h. Por outro lado, não coincide com os resultados de Yannakopoulos \& Serveni-Gousi (1987), que verificaram menor peso para ovos postos e coletados pela manhã.

Independentemente dos tratamentos, o PESOVO tende a aumentar com a idade das aves, seguindo a equação quadrática: $\hat{\mathrm{Y}}=15,741+2,0355 \mathrm{x}-0,0173 \mathrm{x}^{2}$ $\left(\mathrm{R}^{2}=0,978\right)$, sugerindo que galinhas mais velhas produzem ovos mais pesados (Yannakopoulos \& Serveni-Gousi, 1987).

Para GE, as interações tratamentos x coletas e tratamentos x semanas fizeram com que o efeito não fosse o mesmo para as fontes de variação. Entretanto, em razão do efeito altamente significativo para tratamentos, a GE aumentou com o horário da coleta e foi, na maioria das vezes, maior para as aves arraçoadas às $11 \mathrm{~h}$ e às $15 \mathrm{~h} 30$, que para as demais, independentemente de coletas e semanas (Tabela 3).
A GE diminuiu com a idade das aves (Tabela 4 e Figura 2), de forma similar ao observado por Yannakopoulos \& Serveni-Gousi (1987), que verificaram que ovos coletados à tarde apresentaram maior gravidade específica que aqueles coletados pela manhã e que galinhas mais velhas produzem ovos com GE menor e, conseqüentemente, com casca mais fina.

Farmer \& Roland Sr (1983), observando os níveis de Ca nos diferentes segmentos do aparelho digestivo, constataram que reprodutoras alimentadas à tarde, tiveram mais $\mathrm{Ca}$ disponível durante o estágio de calcificação do ovo que as reprodutoras alimentadas pela manhã. Neste trabalho, parece que a disponibilização do Ca para a glândula calcífera foi menor nas aves arraçoadas às $6 \mathrm{~h} 30 \mathrm{e}$ dual no momento da formação da casca, o que confirma os estudos de Brake (1985), que constatou que a GE foi menor para ovos postos e coletados de $9 \mathrm{~h}$ às $13 \mathrm{~h}$ e maior para ovos postos e coletados de $15 \mathrm{~h}$ às $19 \mathrm{~h}$, razão pela qual o autor sugere a existência de variação na GE dos ovos postos no decorrer do dia, em função do acesso ao alimento.

A GE foi beneficiada $(\mathrm{p} \leq 0,05)$ com o arraçoamento mais tardio. Resultados semelhantes foram constatados por Farmer \& Roland Sr (1983), Bootwalla et al. (1983), Brake (1985), Yannakopoulos \& TserveniGousi (1987), Brake (1988) e Harms (1991b). A menor GE para ovos de reprodutoras postos e coletados de $9 \mathrm{~h}$ e $13 \mathrm{~h}$, associada ao menor PESOVO, observado por Brake (1985), se assemelha aos resultados médios obtidos neste trabalho, em que se constatou aumento na GE nos ovos postos e coletados a partir de $9 \mathrm{~h}$, indicando que ovos coletados nas primeiras horas do dia apresentam cascas mais finas. Estes dados confirmam os de Roland Sr \& Harms (1974), que verificaram que a GE e o PESOVO estão relacionados ao horário de postura e que a melhoria na qualidade da casca dos ovos postos à tarde não depende da redução do peso dos mesmos.

Verifica-se que geralmente ovos mais pesados indicam menor GE. No entanto, os dados revelam que igual PESOVO necessariamente não está associado à igual GE. $\mathrm{O}$ inverso é verdadeiro, pois semelhantes GE não necessariamente apresentaram o mesmo PESOVO, corroborando as observações de Roland Sr \& Harms (1974).

Considerando as médias de PESOVO e GE das aves que receberam arraçoamento dual, estes dados são similares aos obtidos por Brake (1988), que obser- 
vou que o arraçoamento mais tarde do dia apresentou considerável aumento no PESOVO com redução na GE, fato atribuído ao aumento do peso da gema e do albúmen. Portanto, outro fator além de PESOVO passou a influenciar na qualidade da casca, que possivelmente tenha sido o efeito do horário de arraçoamento.

As aves com arraçoamento dual, embora tenham recebido $50 \%$ da alimentação à tarde, não responderam à GE do mesmo modo que as aves arraçoadas às $11 \mathrm{~h}$ e às $15 \mathrm{~h} 30$. Porém, esse tratamento favoreceu o aumento do PESOVO, possivelmente por disponibilizar nutrientes para maior síntese de gema e albúmen, o que confirma os estudos de Cave (1981), que verificou vantagem no esquema de alimentação mais vezes ao dia, em razão do aumento na produção de ovos mais pesados durante o período crítico, nas primeiras semanas de postura, e maior média de PESOVO para o período geral. Os resultados de Samara et al. (1996) também foram semelhantes, pois confirmaram que a mudança do horário de alimentação para a tarde não foi efetiva nas aves submetidas ao estresse de calor. No entanto, a alimentação em duas refeições (50\% da ração às $7 \mathrm{~h}$ e $50 \%$ às $18 \mathrm{~h}$ ) foi mais eficiente, como no presente trabalho.

O peso do ovo aumentou com a idade das reprodutoras, como descrito por O'Sullivan et al. (1991), que verificaram a mesma resposta para peso do povo. Os autores também constataram aumentos para peso do albúmen, da gema e da casca, de modo que o elevado peso do albúmen foi atribuído ao incremento no conteúdo de água e da casca e gema, ao acúmulo de matéria seca.

\section{Conclusões}

Os sistemas de arraçoamento mais tardios provocaram atraso e diluição da postura das reprodutoras ao longo do dia.

O sistema de arraçoamento dual pode ser uma alternativa ao sistema de arraçoamento único praticado nas primeiras horas do dia.

\section{Literatura Citada}

BOOTWALLA, S.M.; WILSON, H.R.; HARMS, R.H. Performance of broiler breeders on different feeding systems. Poultry Science, v.62, n.12, p.2321-2325, 1983.
BOOTWALLA, S.M.; WILSON, H.R.; HARMS, R.H. Plasma calcium and phosphorus levels of broiler breeders with different feeding schedules. Poultry Science, v.39, n.2, p.391-398, 1989.

BRAKE, J. Relationship of egg weight, specific gravity, and shell weight to time of oviposition and feeding in broiler breeders. Poultry Science, v.64, n.11, p.2037-2040, 1985.

BRAKE, J. Relationship of time and strain to egg shell quality and hatchability in broiler breeders. Poultry Science, v.67, n.4, p.538-543, 1988.

CAVE, N.A. Effect of diurnal programs of nutrient intake on performance of broiler breeder hens. Poultry Science, v.60, n.6, p.1287-1292, 1981 .

FARMER, M.; ROLAND SR, D.A. Calcium metabolism in broiler breeder hens. 2. The influence of the time of feeding on calcium status of the digestive system and eggshell quality in broiler breeders. Poultry Science, v.62, n.3, p.465-471, 1983.

HARMS, R.H. The influence of changing time of feeding on performance of broiler breeder hens. Poultry Science, v.70, n.8, p.1695-1698, 1991b.

O’SUllivan, N.P.; DUNNINGTON, E.A.; SIEGEL, P.B. Relationships among age of dam, egg components, embryo lipid transfer, and hatchability of broiler breeder eggs. Poultry Science, v.70, n.10, p.2180-2185, 1991.

ROBINSON, F.E.; ROBINSON, N.A.; HARDIN, R.T. The effects of 20-week body weight and feed allocation during early lay on female broiler breeders. Journal of Applied Poultry Research, v.4, n.2, p.203-210, 1995.

ROLAND SR, D.A.; HARMS, R.H. Specific gravity of eggs in relation to egg weight and time of oviposition. Poultry Science, v.53, n.4, p.1494-1498, 1974.

RUTZ, F. Efeito da alimentação e nutrição da matriz sobre a qualidade dos pintos. In: MINI-SIMPÓSIO COLÉGIO BRASILEIRO DE NUTRIÇÃO ANIMAL, 7., 1992, Campinas. Anais... Campinas: Colégio Brasileiro de Nutrição Animal, 1992. p.39-56.

SADIA. Agropastoril Catarinense Ltda. Manual Arbor Acres. Faxinal dos Guedes, SC: Sadia, 1993. 26p.

SAMARA, M.H.; ROBBINS, K.R; SMITH, M.O. Interaction of feeding time and temperature and their relationship to performance of the broiler breeder hen. Poultry Science, 75, n. 1, p.34-41, 1996.

STATISTICAL ANALYSES SYSTEM - SAS. System for microsoft Windows. release 6.12, Cary:1996. CD-ROM.

SUMMERS, J.D. Feeding and management of broiler breeders. In: CONGRESSO LATINOAMERICANO DE AVICULTURA, 10., 1987, Buenos Aires. Proceedings... Buenos Aires: ALA/ CAPIA, 1987, p.29-37.

WILSON, H.R.; MATHER, F.B.; BRIGMON, R.L. et al. Feeding time and temperature interactions in broiler breeders. Poultry Science, v.68, n.5, p.608-616, 1989.

YANNAKOPOULOS, A.L.; TSERVENI-GOUSI, A.S. Relationship of parents' age, hatching egg weight, and shell quality to day-old chick weight as influenced by oviposition time. Poultry Science, v.66, n.5, p.829-833, 1987. 